\title{
GEOSUL
}

\section{A ESTRUTURA ESPACIAL DOS FRAGMENTOS DE VEGETAÇÃO NATIVA DA BACIA HIDROGRÁFICA DO RIO ARAGUARI EM MINAS GERAIS}

\author{
Laís Naiara Gonçalves dos Reis ${ }^{1}$ \\ Luiz Nishyama ${ }^{2}$
}

\begin{abstract}
Resumo: Os elementos que compõem a paisagem (fragmentos e matriz) são objetos de estudo da Ecologia de Paisagens. Por meio das técnicas de geoprocessamento é possível analisar quantitativamente o tamanho, a forma, o número, o tipo e a configuração dos patches. A área de estudo dessa pesquisa é a Bacia Hidrográfica do Rio Araguari-MG que está localizada na região do Triângulo Mineiro e Alto Paranaíba, na parte ocidental do estado de Minas Gerais, ocupando uma área de $22.146,23 \mathrm{~km}^{2}$. As imagens utilizadas para extração das áreas de vegetação florestal nativa dessa bacia são do sensor OLI/Landsat 8 que apresenta resolução espacial de 30 metros. Como resultado, a área de estudo possui 149.521 unidades de fragmentos florestais de vegetação nativa, com no mínimo $900 \mathrm{~m}^{2}$. As métricas em Ecologia de Paisagens avaliadas são referentes à forma, ao tamanho, à densidade de borda, ao comprimento da borda e à área central para cada classe de fragmento: muito pequeno, pequeno, médio e grande. $\mathrm{O}$ fragmento de vegetação nativa com maior área é a Unidade de Conservação de Galheiros.
\end{abstract}

Palavras-chave: Ecologia de Paisagens; Cerrado; Fragmentação; Vegetação nativa

\section{THE SPECIAL STRUCTURE OF ARAGUARI'S RIVER BASIN PATCHES IN MINAS GERAIS}

\begin{abstract}
The elements that make up the landscape (fragments and matrix) are subjects of the study into Landscape Ecology through geoprocessing techniques it is possible to quantitatively analyze the size, shape, number, type and configuration of ecosystems (patches). The study area of this research is the river basin of the Rio Araguari-MG that is located in the region of Triangulo Mineiro and Alto Paranaíba, in the western part of the state of Minas Gerais, covering an area of 22,146.23 square kilometers. The images used for extracting the areas of native forest vegetation of this basin are the sensor used in this process was the Oli / Landsat 8 which has a spatial resolution of 30 meters. As a result, the study area has 142,492.00 units with at least $900 \mathrm{~m}^{2}$, the metrics evaluated Landscape Ecology refer to the shape, size, edge density, length of edge and core area for each fragment: very small small, medium and large.
\end{abstract}

Keywords: Landscape Ecology; Cerrado; Fragmentation; Native vegetation.

\footnotetext{
${ }^{1}$ Professora Dra. Titular da Universidade Estadual de Goiás do curso de Geografia. Email: geografalais2013@gmail.com

${ }^{2}$ Professor Dr. Titular da Universidade Federal de Uberlândia. Email: luiz.nishiyama@gmail.com
} 


\section{A ESTRUTURA ESPACIAL DE LOS FRAGMENTOS DE VEGETACIÓN NATIVA DE BACIA HIDROGRÁFICA DEL RÍO ARAGUARI EN MINAS GERAIS}

Resumen: Los elementos que componen una región (fragmentos y matriz) son objetos de estudio de la Ecología de Paisajes. Por medio de las técnicas de geoprocesamiento y análisis cuantitativo del tamaño, forma, número, tipo y configuración de los parches. Un área de estudio de la investigación en una Bacia Hidrográfica del Río Araguari-MG que está localizada en la región del Triángulo Mineiro y Alto Paranaíba, en la parte occidental del estado de Minas Gerais, ocupando una superficie de $22.146,23 \mathrm{~km}^{2}$. Como imágenes utilizadas para la extracción de las áreas de vegetación florestal nativa esta bacia son sensores OLI / Landsat 8 que presentan la resolución espacial de 30 metros. Como resultado, un área de estudio tiene 149.521 unidades de fragmentos flores de vegetación nativa, con no mínimo de $900 \mathrm{~m}^{2}$. Como métricas en Ecologia de Paisagens avaliadas son referentes a forma, tamaño, densidad de borda, longitud de borde y área central para cada clase de fragmento: muy pequeño, pequeño, médio y grande. $\mathrm{O}$ fragmento de vegetación nativa con mayor área es una Unidad de Conservación de Galheiros.

Palabras clave: Ecologia de Paisagens; Cerrado; Fragmentação; Vegetación nativa.

\section{INTRODUÇÃO}

Os estudos de conservação e conectividade dos habitats é uma temática urgente, diante dos problemas ambientais do século XXI, em que o foco é a riqueza da biodiversidade na paisagem. Essa riqueza pode ser preservada nos habitats que são componentes estruturais do mosaico de paisagens. A natureza ficou a mercê da exploração compulsiva do Homem por, pelo menos, quatro séculos, o que levou a organização e ao desenvolvimento do mundo contemporâneo, onde as desigualdades e mazelas sociais estão escancaradas pelos continentes. A partir da década de 60, começaram os movimentos autônomos pela busca de melhoria de condições de vida, de trabalho, do direito da mulher, e inclusive contra a forma em que o meio ecológico estava condicionado pela lógica econômica mundial.

Como agravante deste cenário de exploração dos recursos naturais, a biodiversidade encontra-se ameaçada. A riqueza e a abundância de espécies dependem das características estruturais dos fragmentos de vegetação nativa (METZGER, 2000). O sucesso de uma espécie (adaptação) em um determinado meio é fruto da interação com os fatores abióticos e bióticos sob um limite de tolerância. Isto é, cada espécie possui um nicho ecológico, onde ela enfrenta 
fatores limitantes, cuja diversidade genética é muito importante para que os organismos possam responder e se adaptar às mudanças ambientais. Quanto maior for a área de refúgio natural para os organismos, mais chances eles possuem de se dispersarem e aumentarem a variabilidade genética da biodiversidade.

A avaliação da qualidade dos habitats (manchas de vegetação nativa) da paisagem pode ser feita por parâmetros estabelecidos pela Ecologia de paisagens. Essa utiliza o potencial de investigação regional para analisar os padrões ecológicos espaciais. Especificamente, nos estudos dos habitats fragmentados é possível fazer o levantamento da métrica dos patches (manchas) de vegetação nativa (FVN), isto possibilita a avaliação da susceptibilidade ambiental dessas áreas em relação à perda da biodiversidade, isto é, auxilia no processo de separação das zonas onde a fauna e a flora estão vulneráveis à extinção.

A aliança entre o geoprocessamento e a Ecologia de paisagens é uma ferramenta importante para auxiliar na compreensão de padrões espaciais ecológicos. Desta maneira, é preciso estabelecer a escala espacial e temporal com afinco. Em noções e conceitos de tratamento de dados espaciais, a investigação pode perpassar por três linhas: modelagem cartográfica, matemática e métodos estatísticos. Dale e Fortin (2014, p. 1, tradução nossa) detalharam essas linhas para o tratamento em dados ecológicos: “[...] descrever e testar a estrutura espacial da paisagem, a extrapolação e interpolação espacial desta, regredir e simular os processos da paisagem, analisar a interação destes, e realizar a modelagem considerando as escalas espaço-temporal do objeto de estudo".

O aparato geotecnológico apresenta diversos aplicativos computacionais que permitem fazer a análise métrica descritiva das estruturas da paisagem. Em Ecologia de paisagens, “[...] esses programas caracterizam a fragmentação de uma paisagem, fornecendo valores quantitativos de extensão de área e de distribuição espacial dos diferentes tipos de fragmentos que compõem uma paisagem" (HESSBRURG et al., 2000 apud PIROVANI et al. 2012).

Os dados utilizados em Ecologia da paisagem podem ser espaciais ou não. As análises espaciais referem-se à relação espacial entre diferentes ecossistemas ou elementos presentes na paisagem (fragmentos e matriz), ou seja, é possível analisar quantitativamente o tamanho, forma, número, tipo e configuração dos FVN (patches). 
Para Forman e Gordon (1986), as métricas são importantes informações da paisagem, além de serem base para cálculo de alguns índices, tais quais:

- Índice de Densidade e Tamanho: são índices referentes ao número de fragmentos, tamanho médio dos mesmos, desvio padrão e coeficiente de variação de tamanho (MCGARIGAL et al., 2002) .

- Índice de bordas: são áreas de transição entre as unidades de paisagens distintas, e esse índice auxilia no levantamento da quantidade de áreas que estão sobre o efeito de bordas (HOLLAND, 1988 apud METZGER, 1999).

- Índice de forma: a forma de um fragmento influencia diretamente na quantidade de área sobre efeito de borda, em que o perímetro de mancha está diretamente ligado à forma do mesmo (VOLOTÃO, 1998).

- Índices de proximidades: refere-se à distância do vizinho mais próximo. Esse índice permite avaliar o grau de isolamento dos fragmentos (VOLOTÃO, 1998; FORMAN e GORDON, 1986).

- Índice de Área Central: permite refletir sobre a configuração de uma paisagem, e depende dos outros índices (índice de borda e forma). Avalia se o fragmento apresenta influencias mínimas das externalidades, uma vez que, essa área separa a área de borda (VOLOTÃO, 1998).

Forman (2006, p. 45-46) estabeleceu duas teorias de análise para os patches, Large or a small patches - LOS e single large or several small patches- SLOSS. Essas teorias são importantes para o planejamento do mosaico da paisagem, isto é, elaboração de planos de aptidão e conservação para uma determinada área. Elas trazem reflexões importantes sobre o tamanho ideal para os fragmentos, a quantidade e a localização desses na unidade de planejamento e, consequentemente, a possibilidade de preservação dos fluxos de energia e matéria do meio.

Os pacthes apresentam formas diversificadas na paisagem, devido, sobretudo, às conversões dos fragmentos para os demais usos da matriz. Cada formato apresenta perdas na conservação dos habitats, por conta do aumento das externalidades sobre a área do FVN 
(FORMAN, 2006, p. 117). A Figura 1 apresenta tipos de formatos para classificação dessas unidades quanto à sua forma geométrica.

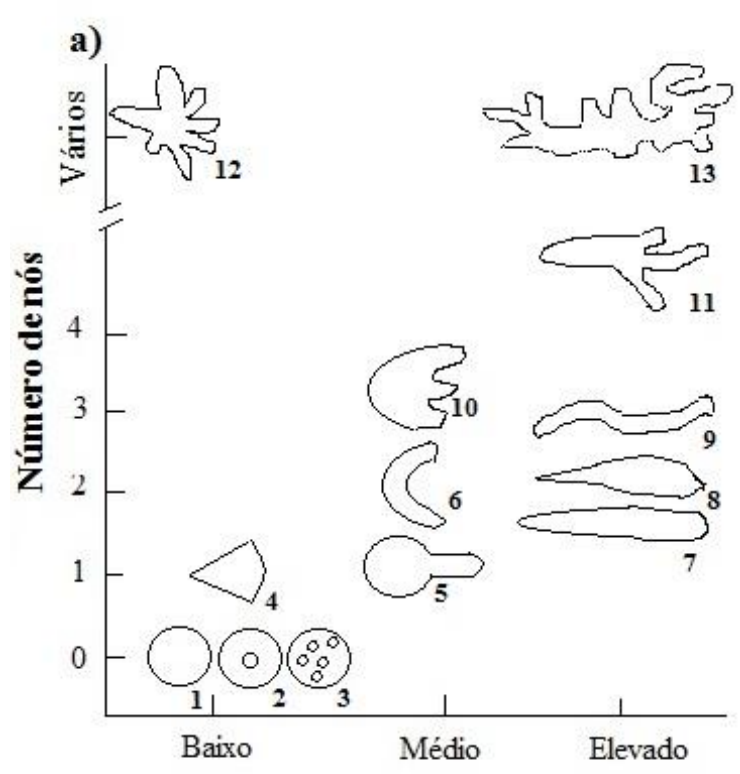

Alongamento

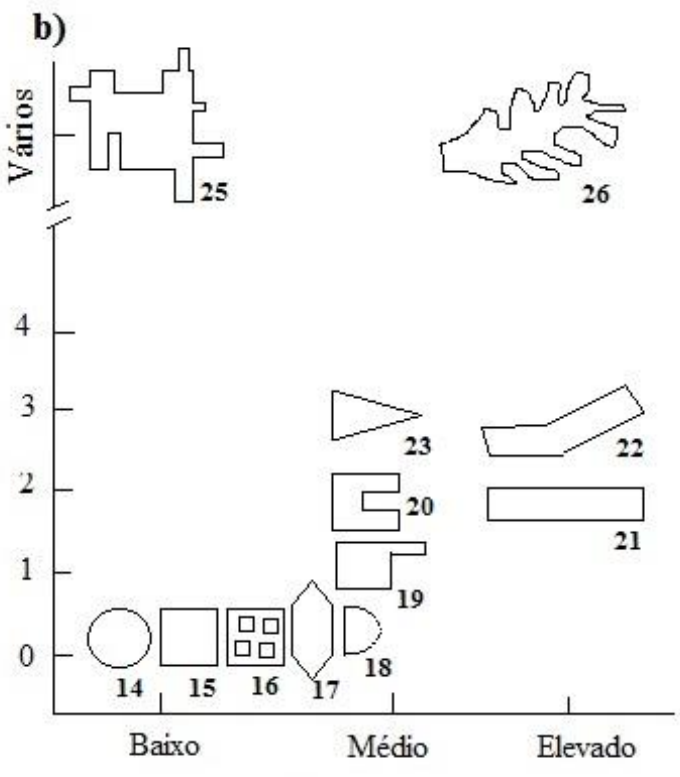

Alongamento

Figura 1 : Formato dos patches encontrados na paisagem fragmentada. Fonte: FORMAN, 2006, p. 117

Sobre a Figura 1, os números 1, 2, 3, 14 apresentam formato round (circular). Amorfhic para as unidades com formas irregulares, 13. Stream-lined patches para aqueles que são estruturas mais alongadas e lineares, como 7, 8 e 9. Indented patches (estruturas endentadas), removal of a portion along one side, exemplo 20, ou seja, remoção de uma parte de um lado do fragmento. Sponge-like or perforated paches (fragmentos perfurados), número 2, 3 e 16. Donut shape or ring patches (formato de anéis-toro, isto é, com uma única perforação), 12. Outros formatos de fragmentos não são tão comuns como, por exemplo, triângulos, cones, quadrados, etc. O formato desses fragmentos florestais de vegetação nativa (FVN) varia em conformidade com o terreno (declividade, aptidão agrícola, entre outros). 
As métricas em Ecologia de paisagens para avaliação da forma, disposição espacial e tamanho dos fragmentos assumem o círculo como a figura ideal para o mesmo, pois é a figura geométrica com menor perímetro (borda do fragmento). Quanto mais distante desta figura, mais irregular será a forma do patch. Quanto maior for a borda de um fragmento maior será sua área em contato com as externalidades provenientes da matriz (demais usos).

Farina (2006) apresentou algumas fórmulas para as métricas espaciais. A Equação 1 descreve a relação entre perímetro e área do fragmento de vegetação nativa

$$
\frac{L}{S}
$$

Em que $\mathrm{L}=$ Perímetro e $\mathrm{S}=$ Área. A relação entre essas duas variáveis indica a qualidade do habitat, uma vez que o quanto maior for o perímetro e menor for a área de um fragmento indica que o mesmo apresenta formato irregular. Já que a figura geométrica que apresenta menor perímetro é o círculo. Dessa forma, a correlação entre perímetro e área está descrito na Equação 2.

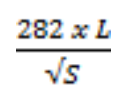

Outro fator importante é a densidade de fragmentos de vegetação nativa no mosaico da paisagem (Equação 3). Se a densidade de fragmentos for representativa significa que há bastante nós (patches) para o estabelecimento de estratégias ambientais para a conectividade desses habitats.

$$
\frac{N i}{A}
$$

Para avaliação do tamanho e quantidade de manchas pela paisagem, o índice de Hulshoff (1995)- S1 mostra que valores altos indicam a presença de muitos fragmentos com áreas pequenas, Equação 4. 


$$
\frac{1}{N i} \sum \frac{L i}{S i}
$$

Em que Ni é o número de patches da área de estudo, Li é o perímetro e Si é a área total dos fragmentos.

Para saber se existe simetria na forma dos fragmentos de uma determinada área utiliza-se o índice 2 de Hulshoff (1995), Equação 5.

$$
\frac{1}{N i} \sum \frac{L i}{4 \sqrt{S i}}
$$

O tamanho médio dos fragmentos (Mean Patch Size-MPS) é estimado pela equação 6.

$$
\frac{\sum_{j=1}^{n} a i j}{n i}
$$

Em que ni é o número de patches e aij é a área de cada patch.

Para encontrar a área do maior fragmento da matriz (Largest Patch Size- LPS), utiliza-se a equação 7, em que A é a área da paisagem.

$$
\frac{\operatorname{Max}(a i j)}{A}
$$

Este trabalho tem como objetivo avaliar o FVN da BHRA por meio da métrica dos patches. Estes estudos são importantes para a Gestão da Conservação da Conectividade dos 
habitats (Connectivity Conservation Management), para a compreensão da distribuição espacial dos fenômenos e gerir as atividades do espaço, reduzindo as ameaças à biodiversidade local e regional. Nessa perspectiva, assumiu-se uma escala regional (22.146,23 $\mathrm{km}^{2}$ ) que possibilitou avaliar quantitativamente e qualitativamente os remanescentes de Cerrado da área de estudo.

\section{Procedimentos técnicos operacionais}

Para avaliar a metodologia investigativa das paisagens fragmentadas do bioma Cerrado foi utilizado um recorte espacial que é a BHRA, que está localizada na região do Triângulo Mineiro e Alto Paranaíba, na parte ocidental do estado de Minas Gerais, ocupando uma área de 22.146,23 $\mathrm{km}^{2}$. Essa está localizada entre as coordenadas $18^{\circ} 20^{\prime}$ e $20^{\circ} 10^{\prime}$ de latitude sul e $46^{\circ} 00^{\prime}$ e $48^{\circ} 50^{\prime}$ de longitude oeste. Abrange as áreas de vinte municípios (alguns parcialmente): Araguari, Araxá, Campos Altos, Ibiá, Indianópolis, Iraí de Minas, Nova Ponte, Patrocínio, Pedrinópolis, Perdizes, Pratinha, Rio Paranaíba, São Roque de Minas, Sacramento, Santa Juliana, Serra do Salitre, Tapira, Tupaciguara, Uberaba e Uberlândia.

\section{Mapeamento dos fragmentos de vegetação nativa da Bacia Hidrográfica do Rio Araguari - Minas Gerais}

Os fragmentos de vegetação nativa mapeados estão inseridos nas seguintes fitofisionomias: a mata ciliar e a de galeria, o cerradão e o cerrado sentido restrito. O sensor utilizado neste processo foi o OLI/Landsat 8 que apresenta resolução espacial de 30 metros. Portanto, não foi possível extrair as áreas de preservação permanente (APP). As cenas utilizadas estão descritas no Quadro 1. Para a extração das informações utilizou-se o software SPRING 5.2 onde foi realizada a classificação supervisionada, aplicando o processo semiautomático, isto é, agrupamento dos pixels semelhantes por meio do processo de segmentação, área de pixel 15 e similaridade 18. Este processo caracteriza-se pelo 
reconhecimento de padrões de alvos homogêneos distinguindo áreas que apresentam comportamentos espectrais diferenciados na superfície, com a eficiência do algoritmo e a capacidade de reconhecer as feições do usuário.

Quadro 1- Identificação das cenas utilizadas no mapeamento de 2014.

\begin{tabular}{|l|l|}
\hline Órbita/ponto & Data \\
$219 / 73$ & $10 / 03 / 2014$ \\
$220 / 73$ & $12 / 06 / 2014$ \\
$220 / 74$ & $13 / 03 / 2014$ \\
$221 / 73$ & $13 / 03 / 2014$ \\
$221 / 74$ & $13 / 03 / 2014$ \\
\hline
\end{tabular}

O algoritmo computacional aplicado para classificar as cenas do sensor OLI/Landsat 8 foi o Maxver, que classifica pixel por pixel, agrupando as informações de cada pixel em regiões homogêneas. Esta classificação associa cada pixel à classe com maior probabilidade de gerar um pixel com as suas características (JENSEN, 1996). O desempenho e limiar de aceitação do mapeamento estão descritos no Quadro 2.

Quadro 2- Resultados da classificação semiautomática dos fragmentos florestais de vegetação nativa para a Bacia Hidrográfica do Rio Araguari do ano de 2014.

\begin{tabular}{|lc|}
\hline \multicolumn{2}{|l|}{ Classificação semiautomática dos Fragmentos Florestais } \\
Desempenho médio & $90,96 \%$ \\
Abstenção Média & $9,04 \%$ \\
Confusão & $0,00 \%$ \\
Classificador Maxver & \\
Limiar de aceitação & $95 \%$ \\
\hline
\end{tabular}

Após a extração dos fragmentos de vegetação nativa (FVN), seguiu-se a metodologia proposta de Juvanhol et al. (2011), num estudo de fragmentação florestal aplicado em área do 
Estado do Espírito Santo, em que os fragmentos foram agrupados em: muito pequeno $\leq 5 \mathrm{ha}$; pequeno $\geq 5,01$ e $\leq 10$; médio $\geq 10,01$ e $\leq 100$ e grande $\geq 100,01$ ha.

\section{Análises dos fragmentos florestais em relação aos índices estruturais da Ecologia de paisagens}

As análises estabelecidas para os fragmentos foram orientadas pelos índices ecológicos e/ou métricas da paisagem. Para tal, foi utilizado o conjunto de ferramentas computacionais chamado Patch Analyst, uma extensão do software da ESRI, disponibilizada

para download gratuito no sítio http://www.cnfer.on.ca/SEP/patchanalyst/Patch5_1_Install.htm> . As métricas obtidas foram: $\mathrm{CA}=$ Área de Todas as Manchas da Classe (hectares), MPS= Tamanho médio das manchas (hectares), PSCoV= Coeficiente de Variação do Tamanho médio das manchas (porcentagem), $\mathrm{PSSD}=$ Desvio Padrão do tamanho das manchas, $\mathrm{TE}=$ Total de Bordas (metros), ED= perímetro de bordas sobre a área do fragmento (metros/hectares), MSI=Índice de Forma Médio, AWMSI=Índice de Forma média de Área Ponderada.

Sabendo-se da importância da área central (core area) para a preservação e conservação da biodiversidade natural, em função desta ser livre do efeito de bordas (edge effect), estas foram calculadas excluindo as bordas de até 100 metros. A maioria das pesquisas indica que este fenômeno ocorre até esta metragem, podendo ser superiores dependendo do objeto de análise (escala de análise). Rodrigues (1998) fez estudos de 0 à 35 metros e de 35 à 100 metros, a partir da borda para o interior do patch.

\section{Resultados e discussão}

De acordo com o antigo Código Florestal - Lei № 4.771, de 15 de setembro de 1965 em relação à Reserva Legal (RL), as propriedades rurais que não possuíam $20 \%$ dessa área com cobertura vegetal nativa deveriam recuperar essas áreas. Na impossibilidade de alocar essas áreas dentro da mesma sub-bacia hidrográfica o órgão ambiental definiria o local para alocação da mesma. Porém, com a aprovação do novo Código Florestal- LEI No 12.651, de 25 
de maio de 2012 - os empreendimentos que danificaram o meio ambiente até junho de 2008 ficaram desobrigados de reflorestar e recuperar tais áreas. Isto significou uma perda de área dos habitats de FVN, tendo como agravante a mudança da área da RL, que era de $20 \%$. Agora, esta área pode ser computada com as de preservação permanente das propriedades.

Para Pirovani (2010), a fragmentação é caracterizada pela ruptura de uma unidade contínua, em que os elementos menores que apresentam dinâmicas e processos ecológicos distintos da unidade original. Cada mancha (patch) se constitui como ecótopo da paisagem (habitat de um ecossistema). Para estudar a dinâmica e o funcionamento desses fragmentos, se destaca a Ecologia de paisagens.

Nesta paisagem fragmentada, algumas manchas são melhores do que as outras, em função do potencial para manutenção da biodiversidade silvestre. Esta análise pode ser inferida pela probabilidade de sobrevivência e reprodução das populações (ou indivíduos) que as ocupam, isto é, da sua aptidão darwiniana. Na paisagem, considera-se que algumas manchas podem ser boas, favoráveis, e outras ruins, menos favoráveis e ainda, que entre elas alguns habitats são completamente desfavoráveis e negativos. Dessa forma, o risco para um dado indivíduo será proporcional ao tempo que este permanecer em cada um dos tipos de habitat para suas atividades vitais (alimentação, reprodução, excreção etc.).

Ainda sobre a conservação da biodiversidade dos fragmentos de vegetação nativa (FVN), sabe-se que esses habitats estão diretamente ligados com os padrões e fluxos dos processos abióticos. A conservação destas áreas implica, inclusive, na manutenção do regime hidrológico, ciclos de reciclagem dos minerais, balanço da radiação, padrão de ventos da atmosfera e dos movimentos de solos (FORMAN, 2006, p. 48).

O mapeamento dos FVN da BHRA (Figura 2) indicou 149.521,00 patches neste recorte espacial, que juntos somavam 245.200,46 ha de remanescentes florestais (Cerradão e Matas) e remanescentes savânicos (Cerrado sentido restrito) do bioma Cerrado em 2014. Os dados que inferem sobre a qualidade dos habitats da BHRA, para este período, estão organizados na Tabela 1. 


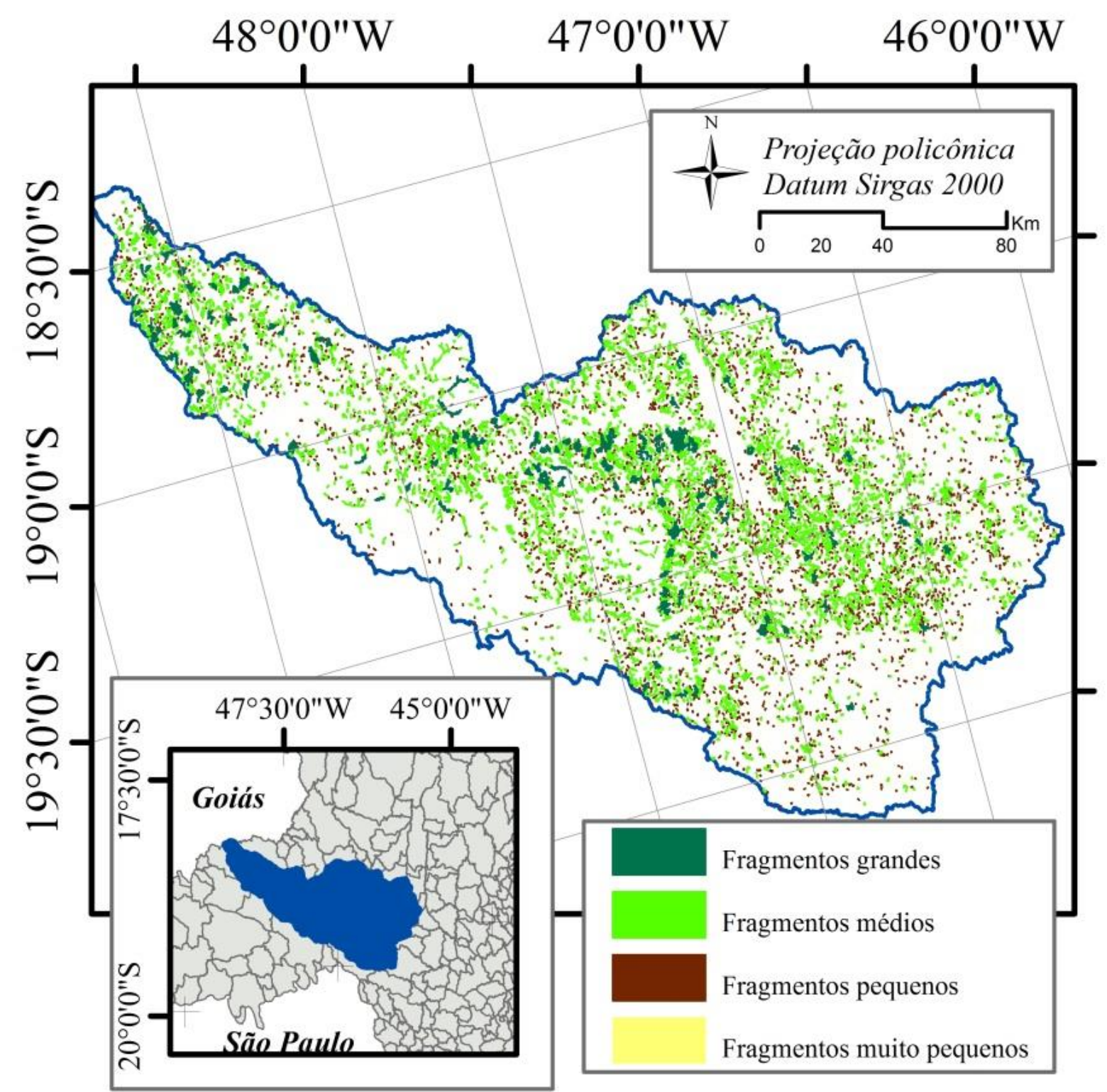

Figura 2- Mapeamento da cobertura florestal nativa da Bacia Hidrográfica do Rio Araguari em Minas Gerais.

Tabela 1: Índices das métricas em Ecologia de paisagens calculados para os fragmentos de vegetação nativa na Bacia Hidrográfica do Rio Araguari em Minas Gerais. 


\begin{tabular}{|c|c|c|c|c|c|c|}
\hline \multirow[b]{2}{*}{ Grupo } & \multirow[b]{2}{*}{ Idices } & \multirow[b]{2}{*}{ Unidades } & \multicolumn{4}{|c|}{ Métricas dos FVN } \\
\hline & & & $\begin{array}{l}\text { MP } \\
(<5 \text { ha })\end{array}$ & $\begin{array}{l}P \\
(5-10 \text { ha })\end{array}$ & $\begin{array}{l}\text { M } \\
\text { (10-100ha) }\end{array}$ & $\begin{array}{l}\text { G } \\
(>100 \text { ha })\end{array}$ \\
\hline Área & CA & Hectares & $77.301,59$ & $31.296,20$ & $107.796,61$ & $28.806,06$ \\
\hline Densid. & MPS & Hectares & 0,54 & 9,07 & 31,24 & 225,05 \\
\hline \multirow[t]{3}{*}{ e tam. } & NUMP & Adimens. & $\begin{array}{l}142.492,0 \\
0\end{array}$ & $3.451,00$ & $3.450,00$ & 128,00 \\
\hline & PSSD & Hectares & 1,01 & 1,84 & 21,74 & 193,36 \\
\hline & PSCoV & Porcent. & 186,96 & 20,29 & 69,58 & 85,92 \\
\hline \multirow[t]{2}{*}{ Borda } & TE & Metros & $\begin{array}{l}36.235 .85 \\
4,31\end{array}$ & $\begin{array}{l}6.993 .202, \\
75\end{array}$ & $\begin{array}{l}17.071 .093, \\
86\end{array}$ & $\begin{array}{l}3.099 .044, \\
66\end{array}$ \\
\hline & ED & $\mathbf{m} / \mathbf{h a}$ & 147,78 & 28,52 & 69,62 & 12,64 \\
\hline \multirow[t]{2}{*}{ Forma } & MSI & Adimens. & 1,20 & 1,90 & 2,49 & 4,56 \\
\hline & AWMSI & Adimensi. & 1,43 & 1,92 & 2,76 & 4,98 \\
\hline
\end{tabular}

Legenda: $\mathrm{CA}=$ Área de Todas as Manchas da Classe (hectares), MPS= Tamanho médio das manchas (hectares), PSCoV= Coeficiente de Variação do Tamanho médio das manchas (porcentagem), $\mathrm{PSSD}=$ Desvio Padrão do tamanho das manchas, $\mathrm{TE}=$ Total de Bordas (metros), $\mathrm{ED}=$ perímetro de bordas sobre a área do fragmento (metros/hectares), MSI=Índice de Forma Médio, AWMSI=Índice de Forma média de Área Ponderada, MPFD=Dimensão Fractal da Forma das Manchas

Do total dos FVN mapeados, na BHRA, a maior parte (142.492 unidades) está inserida na classe de fragmentos muito pequenos (menor que cinco hectares). Juntos representam uma área de 77.301,59 hectares de vegetação nativa fragmentada. Os fragmentos pequenos $(3.451$ unidades) totalizam 31.296,20 hectares. Os fragmentos médios somam 107.796,10 ha e os grandes, apenas 28.806 ha, representados por 128 unidades. O tamanho médio dos fragmentos da bacia é de 61,30 hectares. Conclui-se que a vegetação nativa da BHRA está muito fragmentada e as manchas grandes totalizam somente 12\% de 245.200,46 hectares (Gráfico 1).

Gráfico 1- Distribuição da área de fragmentos florestais de vegetação nativa por classes. 


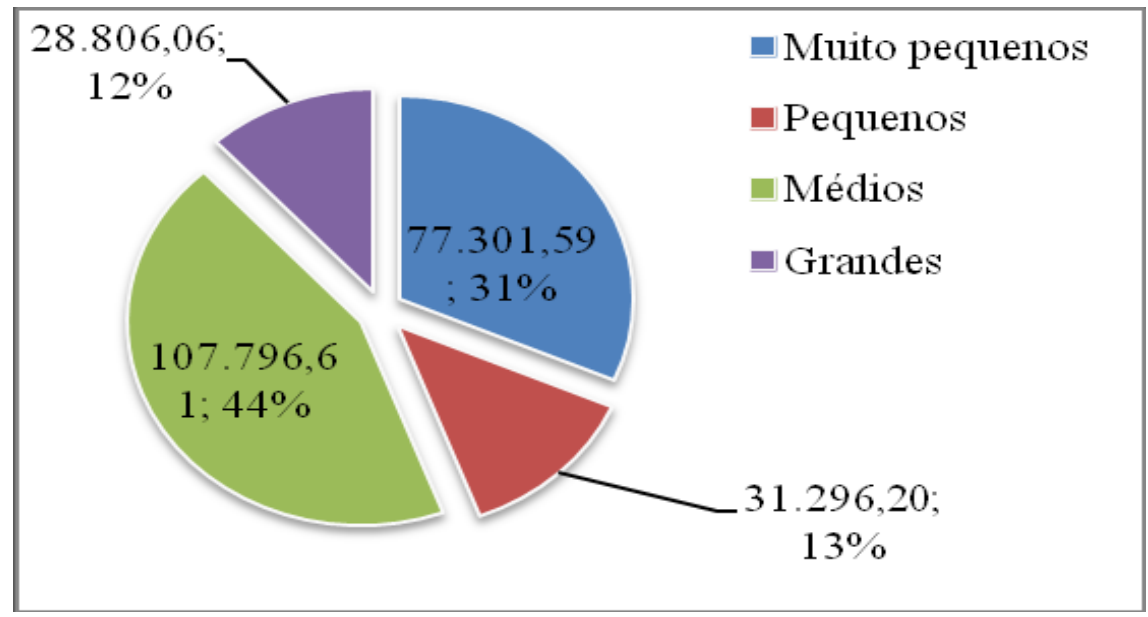

Na BHRA, a maior parte das unidades de patches está inserida nas categorias dos fragmentos muito pequenos e pequenos. Estes são sensíveis ao processo de alteração da paisagem pela atividade humana. Sabe-se que o ideal para a conservação da biodiversidade é a manutenção de fragmentos com áreas maiores, em função da quantidade e qualidade de recursos para a biota, porém o efeito SLOSS (Several Small Patches), também apresenta funcionalidade na paisagem. Essas áreas são consideradas como abrigo temporário para a fauna (fenômeno trampolim) e evitam o isolamento geográfico das mesmas (FORMAN, 2006, pp. 45-46). A espacialização de ilhas muito pequenas dispersas pela matriz, servem de steps stones para a dispersão da fauna. A distância mínima dessas estruturas menores depende de qual espécie é objetivada pela Gestão da Conservação da Conectividade.

A manutenção destes fragmentos menores espalhados pelo mosaico da paisagem é um fato importante, para diminuir o grau de ameaça ao isolamento das espécies. Pois, esse se configura como ameaça de extinção destas em decorrência da competição por recursos e decréscimo da taxa de recolonização em um habitat fragmentado. Para verificar o risco de isolamento geográfico das espécies emprega-se a teoria da percolação que tem como regra básica a premissa que um organismo precisa encontrar pelo menos um recurso ao se movimentar pela matriz (FARINA, 2006, p. 71).

As métricas referentes à borda dos fragmentos são importantes para as tomadas de decisão, no que tange ás estratégias para diminuir a influencia das externalidades provenientes 
da matriz (espécies exóticas, temperatura e umidades diferentes, etc.). A densidade das bordas do fragmento (ED) é a relação do perímetro de bordas com a área do fragmento metros/hectares que revela a relação entre a forma e o tamanho do fragmento. Indicando que os fragmentos grandes da BHRA apresentaram potencialidade maior para conservação da biodiversidade, uma vez que a ED é de 12,64 m/ha. A densidade das bordas é inversamente proporcional à área de cada classe de fragmento (JUVANHOL et al., 2001, p. 359).

As métricas de borda (TE) revelaram que a somatória dos perímetros de todos os fragmentos muito pequenos apresentou o maior valor de borda de todas as categorias (muito pequeno, pequeno, médio e grande), com o montante de 36.235.854,31 metros, isto ocorre em razão da quantidade de unidades dessa categoria na bacia, 142.492,00 unidades. Os fragmentos médios da BHRA apresentaram uma área de borda cerca de $240 \%$ maior do que a área de borda dos fragmentos pequenos, 6.993.202,75 metros.

Os dados da Tabela 1 evidenciaram que os fragmentos estão dispersos na paisagem sem planejamento adequado quanto à forma, apresentando formas irregulares, essas se distanciam da figura ideal, o círculo. Ou seja, é necessário propor um plano com estratégias para incremento da conservação dos habitats para essa bacia em que os patches assumam formatos mais circulares, sem perda da área atual. O indicador que avalia a forma do fragmento (MSI) indica a geometria dos fragmentos que podem ser classificadas em regulares ou irregulares.

Os fragmentos muito pequenos e pequenos da BHRA apresentaram formas mais regulares $(1,2$ e 1,9$)$ diferentemente dos fragmentos médios e grandes $(2,49$ e 4,56). Isso indica que o processo de desmatamento foi desordenado sem sequer orientação adequada dos órgãos competentes IEF e IBAMA, uma vez que estes órgãos não possuem diretrizes para o processo de desmatamento, no que diz respeito à forma ideal para os fragmentos, preocupado em preservar os fluxos ecológicos dos mesmos.

A preocupação com a forma dos patches é fundamental para garantir a qualidade dos habitats, e, por conseguinte a conservação da biodiversidade. O que propicia melhor qualidade nestas áreas da bacia é a variável tamanho, uma vez que, no Brasil não existem normas 
ambientais preocupadas com a forma e determinação locacional eficiente para as reservas de vegetação nativa.

O índice médio ponderado pela área (AWMSI) indica que os fragmentos pequenos são os mais regulares da BHRA. O tamanho do fragmento é importante para a conservação da biodiversidade, porém se o formato deste é irregular significa que a sua borda é maior e, portanto há maior área sofrendo influência do efeito de borda. Ainda sobre o edge effect, segundo Farina (2006), menor é o número de espécies de aves próximas às bordas dos fragmentos. Não existem pesquisas substanciais para mensurar os impactos do efeito de borda para todo o bioma Cerrado. Sabe-se que este fenômeno impacta negativamente a qualidade do habitat devido às mudanças microclimáticas em suas bordas, alterando, assim, a riqueza de diversidade biológica dos patches.

Depois de excluída a área de borda (100 metros) do mapeamento, os FVN da BHRA apresentaram 14.926,50 hectares de área central, isto é, apenas 6\% do total de fragmentos podem estar livres de efeito de borda, conforme indica o Gráfico 2.

Gráfico 2- Relação entre area core e área com efeito de borda dos fragmentos de vegetação nativa da Bacia Hidrográfica do Rio Araguari em Minas Gerais.

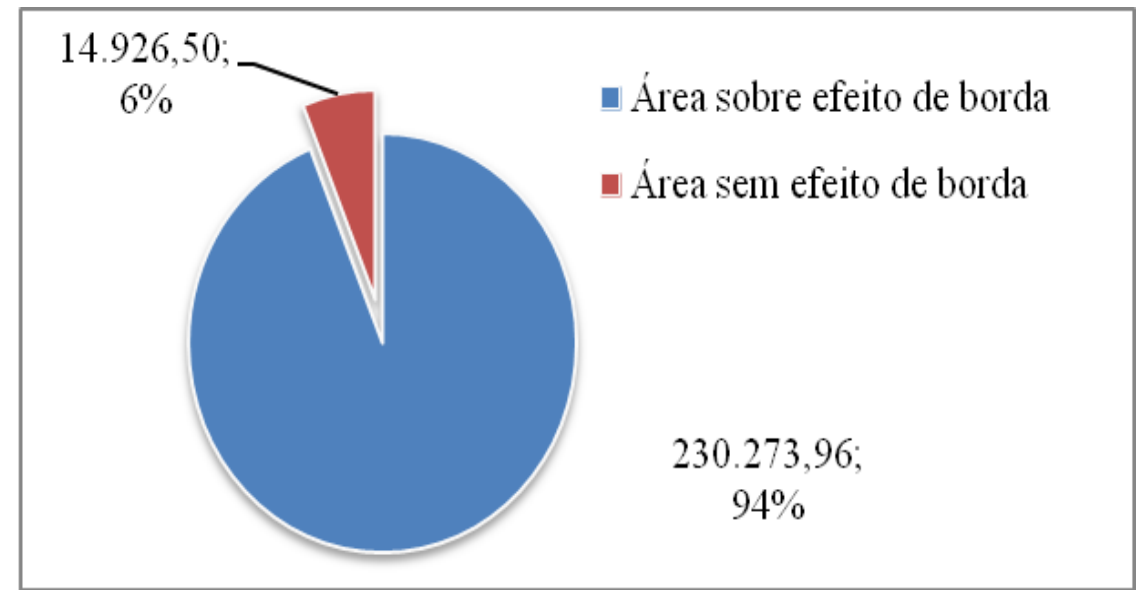

Forman (2006, p. 81) afirmou que o efeito de borda dos FVN em contato com a agricultura são áreas fronteiriças para as pragas e produtos agrícolas. Os processos de erosão do solo também são mais ativos, a entrada de luz nas bordas dos FVN altera a composição florística e 
faunística deste habitat, etc. E neste cenário o Homem atua como espécie importante e agravante desse fenômeno, pois é responsável por aumentar as bordas dos fragmentos, uma vez que ele é o arquiteto deste mosaico de paisagens.

No bioma Cerrado, o efeito de borda nos habitats florestais evidencia as mudanças quanto à composição florística, em decorrência do aumento da taxa da mortalidade dos indivíduos, aumento de cipós e invasão de espécies exóticas, como a Brachiaria ssp. (LAURANCE et al, 2001). A preocupação com a forma dos fragmentos florestais de vegetação nativa é urgente e deve ser objeto de análise para o planejamento ambiental de uma determinada região.

\section{Considerações Finais}

A BHRA apresentava 142.492,00 unidades de fragmentos muito pequenos (menor que cinco hectares), 3.451,00 unidades de fragmentos pequenos (entre 5 e 10 hectares), 3.450 unidades de fragmentos médios (entre 10 e 100 hectares) e 128 unidades de fragmentos grandes (maior que 100 hectares).

Na BHRA, a maioria dos fragmentos remanescentes de Cerrado já sofreu alteração antrópica, portanto, a paisagem é um ambiente herdado, modificado pelo Homem. Apenas $6 \%$ do total de áreas de fragmento florestal de vegetação nativa deste bioma, na área de estudo, podem não apresentar interferência do efeito de borda. Sendo que os fragmentos médios e grandes apresentam formas irregulares, portanto, com bordas extensas. Existem muitos fragmentos pequenos espalhados pela matriz dessa bacia, que são importantes para facilitar o fluxo gênico evitando o isolamento geográfico. Notou-se também a baixa expressividade dos fragmentos maiores.

A Ecologia de Paisagens reúne técnicas e métodos próprios para investigação de padrões e qualidade dos habitats que auxiliam no planejamento ambiental. Quase todo o referencial sobre esse ramo do conhecimento é proveniente da literatura estrangeira. Ainda é um ramo pouco explorado no Brasil. Dessa forma, as teorias não foram pensadas para os 
biomas neotropicais. Surge a necessidade de estabelecer uma linha de pesquisa para testar esses modelos para a realidade do Cerrado, com experimentos em campo e monitoramento da fauna e floral e do efeito de borda sobre os FVN.

\section{Referências bibliográficas}

DALE, Mark R. T.; FORTIN, Marie-José. SAPATIAL ANALYSIS: A guide for ecologists. $2^{a}$ ed. Cambridge. United Kingom, 2014. p.438

FARINA, Almo. Principles and methods in landscape ecology: toward a science of landscape. Holanda: Kluwer Academic Pub, 2006.

FORMAN, M.; GODRON, M. Landscape ecology. New York: Wiley, 1986. 619p.

FORMAN, Richard, T. T. Land Mosaicas: The ecology of landscapes and regions.

Cambriddge University Press. $9^{a}$ ed. 2006. p. 1-604.

FORTIN, M. J; DALE, M. R. T. Spatial Analysis: A guide for ecologists. $2^{\mathrm{a}}$ ed. Universtity of Toronto, 2014. p. 1-438.

LAURANCE, W. F; YENSEN, E. Predicting the impacts of edge effects in fragmented habitats. Biological Conservation. 1991 ;55:77-92.

JENSEN, J.R. Introductory digital image processing. London, Prentice Hall, 1996. P. 316

JUVANHOL, Ronie Silva , Fiedler, Nilton Cesar, Alexandre Rosa dos Santos, Daiani

Bernardo Pirovani , Franciane Lousada Rubini de Oliveira Louzada, Henrique Machado

Dias1, André Luiz Campos Tebaldi. Análise Espacial de Fragmentos Florestais: Caso dos

Parques Estaduais de Forno Grande e Pedra Azul, Estado do Espírito Santo. 2011. Floresta e

Ambiente; v.18. n.4. pp.-353-364. Disponível em:<

http://www.mundogeomatica.com.br/Publicacoes/Artigo27.pdf> Acesso em: out. 2015.

MCGARIGAL, K. et al. FRAGSTATS: Spatial Pattern Analysis Program for Categorical Maps. Computer software program produced by the authors at the University of

Massachusetts, Amherst, 2002. Disponível em

:<http://www.umass.edu/landeco/research/fragstats/fragstats.html > . Acesso em 23 ago. 2011.

Acesso em jan. 2015.

METZGER, Jean. Paul. Como restaurar a conectividade de paisagens fragmentadas. In:

Simpósio de Restauração de Ecossistemas Degradados com Espécies Nativas. São Paulo: Edusp, 2000.

. Estrutura da paisagem e fragmentação: análise bibliográfica. Anais da Academia

Brasileira de Ciências, v.71, n.3-I, p.445-463, 1999.

PIROVANI, Daiani Bernardo. Uso de geotecnologias para estudo da fragmentação florestal com base em princípios de Ecologia da Paisagem. IN: Geotecnologias aplicadas aos recursos naturais. Org. SANTOS, A. S, et al. - Alegre, ES: CAUFES, 2012. Disponível em: $\langle$ http://www.mundogeomatica.com.br/Livro_Geoteconologia_Recursos_Florestal.htm $>$ Acesso em: mai. 2015. 
Fragmentação, florestal, dinâmica e ecologia da paisagem na bacia hidrográfica do rio Itapemirim, ES. Dissertação (mestrado) - Universidade Federal do Espírito Santo. Centro de Ciências Agrárias, 2010.

RODRIGUES, Efraim. Efeito de borda em fragmentos de florestas. Opinião. Londrina: Cadernos da Biodiversidade. Disponível em $:<$

http://www.iap.pr.gov.br/arquivos/File/Publicacoes/Cadernos\%20da\%20Biodiversidade/Cade srnos_da_Biodiversidade_V1n2/CADERNOS_2_VERS.pdf> Acesso em: out. 2015. VOLOTÃO, C.F.S. Trabalho de análise espacial Métricas do Fragstats. Dissertação de Mestrado. São Jose dos Campos: INPE, 1998.

Recebido em abril de 2016.

Aceito em julho de 2017. 